\title{
Topology optimisation for heat conduction problems in 2D with heat transfer boundary condition and heat flux objective function defined on morphing boundaries using BEM
}

\author{
G. Jing ${ }^{1}$, H. Isakari ${ }^{1}$, T. Matsumoto ${ }^{1}$, T. Yamada $^{2} \&$ T. Takahashi ${ }^{1}$ \\ ${ }^{1}$ Nagoya University, Japan \\ ${ }^{2}$ Kyoto University, Japan
}

\begin{abstract}
Topological derivative is a significant and indispensable quantity in topology optimisation processes. It is defined as the limit of the variation of the objective functional caused when an infinitely small part of the material is removed in the domain. Since the topological derivatives have different expressions depending on the boundary conditions on the boundary of the cavity generated by removing the material, the derivation of the topological derivative becomes an important task before proceeding to the topology optimisation. This paper presents a topological derivative expression for two-dimensional heat conduction problems with the objective function of heat flux defined on the morphing boundaries generated through the topology optimisation process. The obtained topological derivative is used to update the distribution of the level set function, which gives the boundary of the material as its iso-surface corresponding to zero value. The effectiveness of the present approach is demonstrated through numerical examples.

Keywords: level set method, topology optimisation, topological sensitivity, heat conduction problems, heat flux, boundary element method.
\end{abstract}

\section{Introduction}

Topology optimisation is a structural optimisation (Bendsøe and Sigmund [1]) approach that can redistribute the layout of material. It has the highest design freedom compared with sizing (Nguyen et al. [2]) and shape optimisation (Sokolowski and Zolesio [3]) methods. Therefore, it has received close attention 
and has been studied extensively so far. Topology optimisation has been developed in many branches, such as the homogenization method (Suzuki and Kikuchi [4], Bendsøe and Kikuchi [5]), solid isotropic material with penalization (SIMP) [6]. Later, Yamada et al. [7] proposed a topology optimisation method using level set method, which can avoid the checkerboard and intermediate density problems existed in the existing methods.

Topology optimisation has been applied to various problems, e.g. mechanics [8], acoustics [9] and heat conduction [10]. Finite element method (FEM) [11] played an important role in analysing the physical phenomena in these problems. Also, the area in which the material vanishes through the optimisation process has been treated as the weak material by giving sufficiently small values to the material properties. This drawback may be solved if the finite element mesh is updated for the actual material domain through the optimisation process, however, this is costly because the FEM mesh generation is costly. In this study, we consider a topology optimisation for two-dimensional steady state heat conduction problems. We use the boundary element method as the computation method to solve the heat conduction problem. The boundary of the material under morphing through the optimisation process is extracted from the iso-surface of the level set function whose value is zero, and the boundary mesh is generated at every updating process. Another feature of the present study is that the objective function for controling the heat flux is also defined on the boundary which emerges through the topology optimisation process. This gives a different expression of the topological derivative from the expression without such objective function. The effectiveness of the proposed expression for the topological derivative and the entire analysis scheme of topological optimisation are demonstrated through some numerical examples.

\section{Formulations}

\subsection{Topology optimisation problem description}

We consider the following optimisation problem where the objective function is defined only with the heat flux on the boundary $\Gamma$ including the boundaries which emerge through boundary updating steps of the optimisation.

$$
\inf _{\phi} I=\int_{\Gamma} f(q) d \Gamma,
$$

subject to

$$
\begin{array}{lll}
\text { D.E.: } & \nabla \cdot(-k \nabla T)=0 & \text { in } \Omega, \\
\text { B.C.: } & T=\bar{T} & \text { on } \Gamma_{T}, \\
& q=-k \frac{\partial T}{\partial n}=\bar{q} & \text { on } \Gamma_{q}, \\
& q=h\left(T-T_{a}\right) & \text { on } \Gamma_{h} .
\end{array}
$$


and

$$
G=\int_{D} \mathscr{H}(\phi(x)) d \Omega-G_{\max } \leq 0,
$$

where $f(q)$ is defined only with the heat flux $q=-k \frac{\partial T}{\partial n}$ on $\Gamma . T$ is the temperature, $n$ is the outward normal direction to $\Gamma, T_{a}$ is the ambient temperature, $k$ is the thermal conductivity, $h$ is the heat transfer coefficient, $G_{\max }$ is the allowable upper limit of the area of the material region $\Omega$, and $\mathscr{H}$ is the characteristic function defined as follows:

$$
\mathscr{H}(\phi(x))= \begin{cases}1 & \phi(x)>0 \\ 0 & \phi(x) \leq 0\end{cases}
$$

The objective functional eqn (1) can be augmented by considering eqn (2) together as the equality constraint, as follows:

$$
\inf _{\phi} J=I(\phi)+\int_{\Omega} w \nabla \cdot(-k \nabla T) d \Omega,
$$

where $w$ is the adjoint variable considered as Lagrange's multiplier.

In order to control the shape change, including the topology change, of the material, we introduce the following level set function $\phi(x)$.

$$
\begin{cases}0<\phi(x) \leq 1, & x \in \Omega \backslash \Gamma, \\ \phi(x)=0, & x \in \Gamma, \\ -1 \leq \phi(x)<0, & x \in D \backslash \Omega,\end{cases}
$$

where $D$ and $\Gamma$ denote the fixed design domain and the boundary of $\Omega$, respectively, as shown in Figure 1.

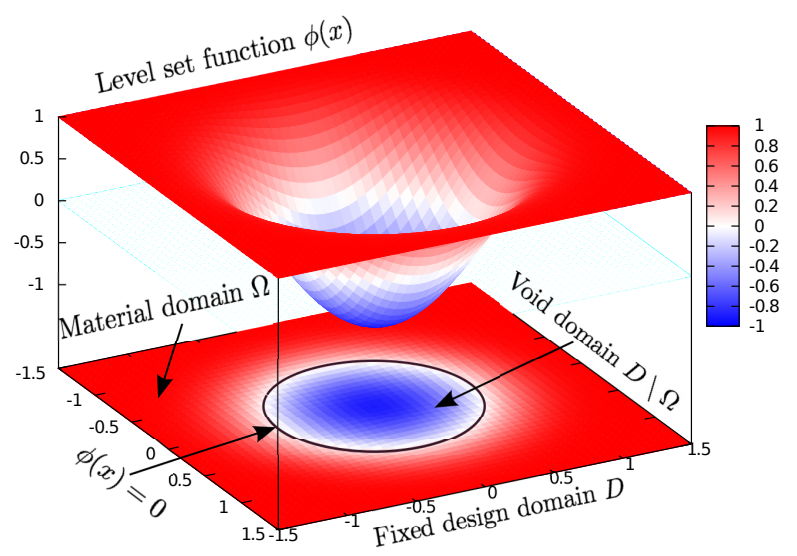

Figure 1: Level set function defined in the fixed design domain $D$. 
In the following, we assume that the level set function $\phi(x)$ changes with respect to a fictitious time $t$ and that its time-derivative changes proportional to the topological derivative $J_{T}^{\prime}$ of the objective functional $J$ accompanied with the area constraint penalty parameter for $0 \leq \lambda \leq 1$. Besides, $\nabla^{2} \phi$, which is related to the mean curvature of the level set function $\phi$, is introduced to control the complexity of the topology of the result. Thus, we consider the following time evolution equation for updating the distribution of the level set function:

$$
\frac{\partial \phi}{\partial t}=K\left(J_{T}^{\prime}-\lambda+\tau \nabla^{2} \phi\right) \quad \text { in } D
$$

where $\tau$ is a parameter that can regularise the complexity of the distribution of $\phi(x)$.

The boundary condition to solve eqn (10) can be set as follows:

$$
\begin{aligned}
& \frac{\partial \phi}{\partial n}=0 \quad \text { on } \partial D \backslash \Gamma_{\text {non }}, \\
& \phi=1 \quad \text { on } \Gamma_{\text {non }},
\end{aligned}
$$

where $K>0$ is a coefficient of proportionality, $\Gamma_{\text {non }}$ represents non-design boundaries of the fixed design domain $D$.

\subsection{Topological derivative}

The augmented objective function $J$ can be rewritten, after integrating by parts, as follows:

$$
J=\int_{\Gamma} f(q) d \Gamma+\int_{\Omega} \nabla w \cdot(-k \nabla T) d \Omega .
$$

We introduce an adjoint field of $w$, which is the solution of the following boundary value problem:

$$
\begin{array}{ll}
\nabla \cdot(-k \nabla w)=0 & \text { in } \Omega, \\
w=-\frac{\partial f}{\partial q} & \text { on } \Gamma_{T}, \\
v=0 & \text { on } \Gamma_{q}, \\
v=h\left(w+\frac{\partial f}{\partial q}\right) & \text { on } \Gamma_{h} .
\end{array}
$$

The objective function suffers from a change, denoted by $\delta J$, when an infinitesimal material region $\Omega_{\varepsilon}$ whose radius is $\varepsilon$, is removed from $\Omega$. Also, on the emerged boundary $\Gamma_{\varepsilon}$ which is the boundary of the cavity generated by removing $\Omega_{\varepsilon}$, heat transfer boundary condition is applied and also the heat flux objective $f(q)$ is considered. After some manipulations, we obtain the leading term as 


$$
\begin{gathered}
\delta J=2 \pi \varepsilon w^{0} h\left(T^{0}-T_{a}\right)+\varepsilon \int_{0}^{2 \pi} f(q) d \theta \\
+\int_{0}^{2 \pi} \frac{\partial f}{\partial q}\left[\frac{k h\left(T^{0}-T_{a}\right)}{k-h \varepsilon \ln \varepsilon}-\frac{k(k-\varepsilon h)}{k+\varepsilon h}\left(T_{, x}^{0} \cos \theta+T_{, y}^{0} \sin \theta\right)\right] d \theta .
\end{gathered}
$$

The topological derivative is defined as:

$$
\begin{aligned}
J_{T}^{\prime}= & \lim _{\varepsilon \rightarrow 0} \frac{\delta J}{2 \pi \varepsilon} \\
= & w^{0} h\left(T^{0}-T_{a}\right)+\lim _{\varepsilon \rightarrow 0} \frac{1}{2 \pi} \int_{0}^{2 \pi} f(q) d \theta \\
& +\lim _{\varepsilon \rightarrow 0} \frac{1}{2 \pi \varepsilon} \int_{0}^{2 \pi} \frac{\partial f}{\partial q}\left[\frac{k h\left(T^{0}-T_{a}\right)}{k-h \varepsilon \ln \varepsilon}-\frac{k(k-\varepsilon h)}{k+\varepsilon h}\left(T_{, x}^{0} \cos \theta+T_{, y}^{0} \sin \theta\right)\right] d \theta .
\end{aligned}
$$

Now, in the present study, we consider the integrand of the objective function of heat flux as:

$$
\begin{aligned}
f(q)= & (q-\hat{q})^{2} \approx\left[k\left(T_{, x}^{0} \cos \theta+T_{, y}^{0} \sin \theta\right)-\hat{q}\right]^{2} \\
= & k^{2}\left[\left(T_{, x}^{0}\right)^{2} \cos ^{2} \theta+\left(T_{, y}^{0}\right)^{2} \sin ^{2} \theta\right]+\hat{q}^{2} \\
& +2 k^{2} T_{, x}^{0} T_{, y}^{0} \cos \theta \sin \theta-2 k \hat{q}\left(T_{, x}^{0} \cos \theta+T_{, y}^{0} \sin \theta\right) \text { on } \Gamma_{\varepsilon},
\end{aligned}
$$

where $\hat{q}$ is the prescribed target heat flux.

$$
\frac{\partial f}{\partial q}=2(q-\hat{q})=2\left[k\left(T_{, x}^{0} \cos \theta+T_{, y}^{0} \sin \theta\right)-\hat{q}\right] \quad \text { on } \Gamma_{\varepsilon} .
$$

Therefore, we have

$$
\begin{aligned}
\lim _{\varepsilon \rightarrow 0} \frac{1}{2 \pi} \int_{0}^{2 \pi} f(q) d \theta & =\lim _{\varepsilon \rightarrow 0} \frac{1}{2 \pi} \int_{0}^{2 \pi}\left\{k^{2}\left[\left(T_{, x}^{0}\right)^{2} \cos ^{2} \theta+\left(T_{, y}^{0}\right)^{2} \sin ^{2} \theta\right]+\hat{q}^{2}\right\} d \theta \\
& =k^{2}\left|\nabla T^{0}\right|^{2}+\hat{q}^{2}
\end{aligned}
$$

Also, using eqn (21), we can get

$$
\begin{gathered}
\lim _{\varepsilon \rightarrow 0} \frac{1}{2 \pi} \int_{0}^{2 \pi} \frac{\partial f}{\partial q}\left[h\left(T^{0}-T_{a}\right)-k\left(T_{, x}^{0} \cos \theta+T_{, y}^{0} \sin \theta\right)\right] d \theta \\
=-2 \hat{q} h\left(T^{0}-T_{a}\right)-k^{2}\left|\nabla T^{0}\right|^{2}
\end{gathered}
$$

Therefore, the topological derivative becomes as

$$
\begin{aligned}
J_{T}^{\prime} & =w^{0} h\left(T^{0}-T_{a}\right)+k^{2}\left|\nabla T^{0}\right|^{2}+\hat{q}^{2}-2 \hat{q} h\left(T^{0}-T_{a}\right)-k^{2}\left|\nabla T^{0}\right|^{2} \\
& =h\left(T^{0}-T_{a}\right)\left(w^{0}-2 \hat{q}\right)+\hat{q}^{2} .
\end{aligned}
$$




\section{Verification of the topological derivative}

The topological derivative given by eqn (24) is verified by comparing the value calculated using eqn (24) with the approximated value calculated by finite difference approximation.

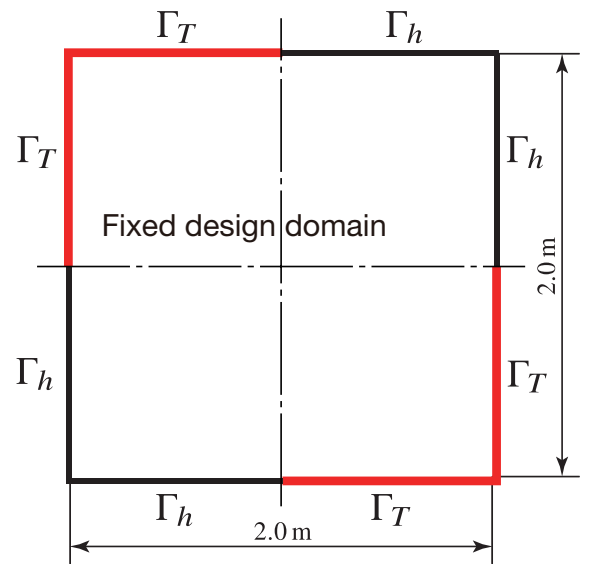

Figure 2: Fixed design domain used to verify the topological derivative expression.

We consider a fixed design domain initially filled with the material entirely in the region of $2.0 \mathrm{~m} \times 2.0 \mathrm{~m}$ as shown in Figure 2 . Temperature boundary conditions are given on the boundaries adjacent to the top-left and bottom-right corners of the design domain with a prescribed temperature $\bar{u}=60^{\circ} \mathrm{C}$, while the heat transfer boundary conditions are prescribed on the boundaries adjacent to the top-right and bottom-left corners, as well as the newly generated boundary appearing in the optimisation updating process, with the ambient temperature $u_{\infty}=40^{\circ} \mathrm{C}$ and the heat transfer coefficient $h=0.002\left[\mathrm{~W} /\left(\mathrm{m}^{2} \cdot \mathrm{K}\right)\right]$. The thermal conductivity of the domain is assumed as $k=1.0[\mathrm{~W} /(\mathrm{m} \cdot \mathrm{K})]$.

We compare the topological derivative values calculated by eqn (24) with the approximate values calculated by the finite difference between the values of the objective functions for the original domain and for the domain from which a small circular hole is deleted.

The objective function is defined as

$$
F=\int_{\Gamma_{h} \cup \Gamma_{\varepsilon}}(q-\hat{q})^{2} d \Gamma
$$

where $\Gamma_{\varepsilon}$ denotes the boundary of the deleted area and $\hat{q}=15.0\left[\mathrm{~W} /\left(\mathrm{m}^{2} \cdot \mathrm{K}\right)\right]$ is the target heat flux.

The boundary of the square fixed design domain is discretized uniformly with $40 \times 4=160$ quadratic conforming boundary elements. Also, $40 \times 40=1600$ 


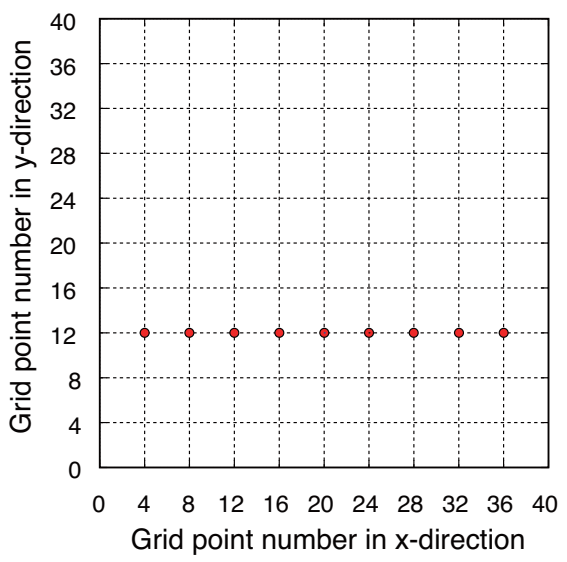

Figure 3: Sample points at which the topological derivatives are verified.

grids, numbered here as $\left(i_{x}^{G}, i_{y}^{G}\right)$ where $i_{x}^{G}=1,2, \cdots, 40$ and $i_{x}^{G}=1,2, \cdots, 40$, are considered in the fixed design domain. The topological sensitivity values are calculated at some of these grid points shown in Figure 3.

The approximate values of the topological derivative is calculated by the following formula:

$$
F_{\text {approx }}^{\prime}=\frac{F_{\text {original }}-F_{\text {hole }}}{2 \pi \varepsilon^{*}},
$$

where $F_{\text {original }}$ and $F_{\text {hole }}$ denote the values of the objective function before and after the hole is created. $\varepsilon^{*}=0.0001[\mathrm{~m}]$ is the radius of the hole, and the boundary of the hole is divided into 32 quadratic elements when $F_{\text {hole }}$ is calculated using the boundary element method. The topological derivative calculated using eqn (24) and those calculated by the finite difference approximation at the sample points of $i_{y}^{G}=12$ are plotted in Figure 4 . We find from the figure that the topological derivative values calculated using the present expression are in very good agreement with those calculated with the finite difference approximation, and the correctness of the derived expression is verified.

\section{Topology optimisation example}

In this example we consider a topology optimisation using the derived topological derivative given by eqn (24). The fixed design domain is a square region of $2.0[\mathrm{~m}] \times 2.0[\mathrm{~m}]$ filled with material as shown in Figure 5. The thermal conductivity is assumed to be $k=1.0[\mathrm{~W} /(\mathrm{m} \cdot \mathrm{K})]$.

Temperature boundary condition is prescribed on the boundaries adjacent to the four corners of the fixed design domain with the temperature $\bar{u}=50^{\circ} \mathrm{C}$. The heat transfer boundary condition is prescribed both on the middle part of each edge of the fixed design domain and on the boundary appearing through the optimisation 


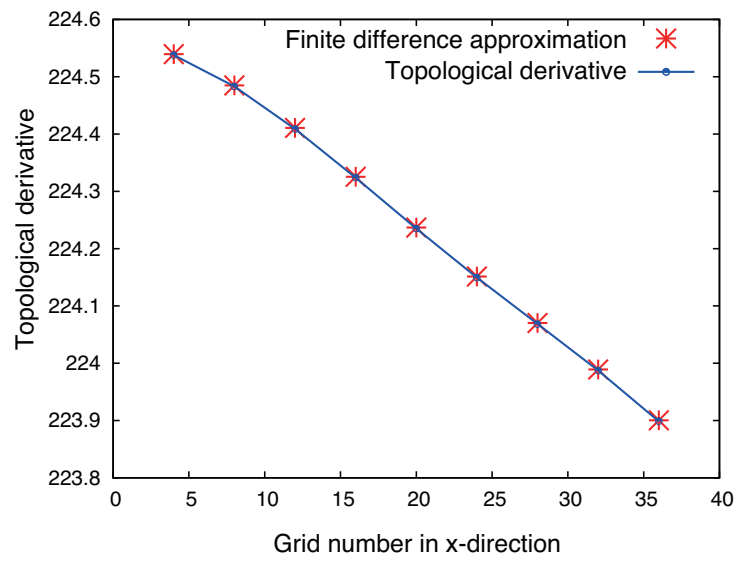

Figure 4: Comparison of the topological derivative values calculated using the analytical expression with those calculated by the finite difference approximation at grid points uniformly arranged along the line: $i_{y}^{G}=12$.

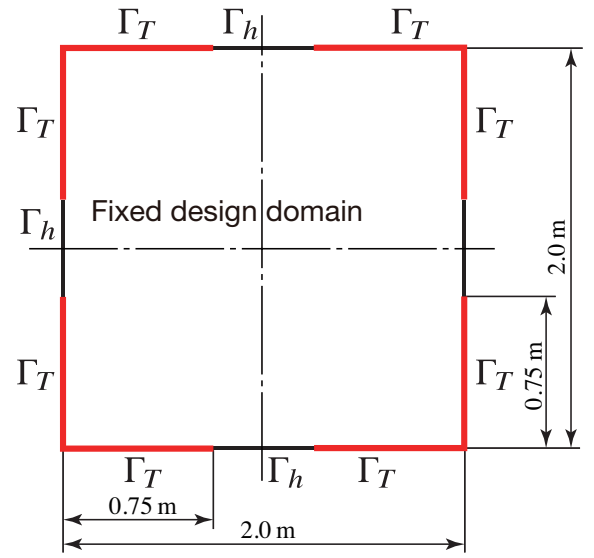

Figure 5: Fixed design domain for topology optimisation example.

process, with the ambient temperature $u_{\infty}=45^{\circ} \mathrm{C}$ and the heat transfer coefficient $h=5.0\left[\mathrm{~W} /\left(\mathrm{m}^{2} \cdot \mathrm{K}\right)\right]$.

In this example the objective function is defined as

$$
F=\int_{\Gamma_{\mathrm{h}}}(q-\hat{q})^{2} d \Gamma
$$

where $\Gamma_{\mathrm{h}}$ denotes all the heat transfer boundary of the design domain. The target heat flux is given as $\hat{q}=5.0\left[\mathrm{~W} /\left(\mathrm{m}^{2} \cdot \mathrm{K}\right)\right]$.

The boundary of the square design domain is discretized uniformly with $40 \times 4=160$ quadratic conforming boundary elements. The area constraint is 


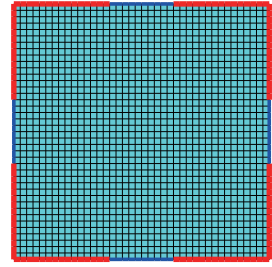

(a)

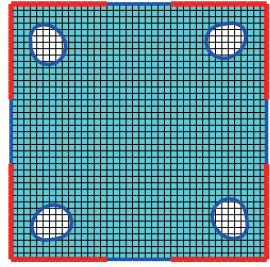

(b)

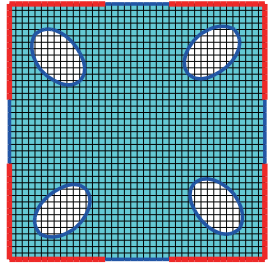

(c)

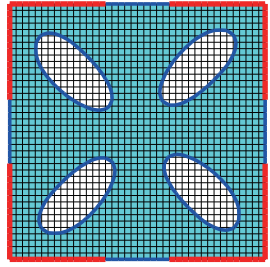

(d)

Figure 6: The obtained configurations: (a) Initial configuration; (b) Configuration at step 20; (c) Configuration at step 40; (d) Optimal configuration.

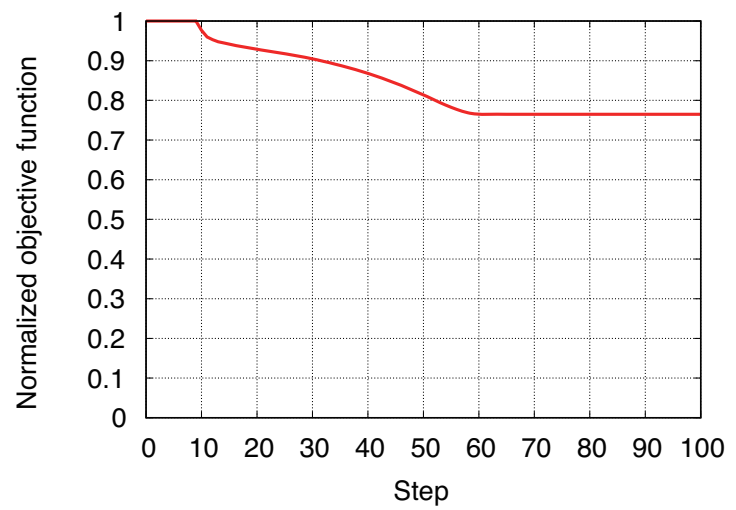

Figure 7: Fixed design domain for topology optimisation example.

set as $G_{\max }=80 \%$ of the area of the fixed design domain. Also, $K=10$ and $\delta t=0.22$, which is the time interval to solve eqn (10), are used. The regularisation term is set as $\tau=5.0 \times 10^{-1}$. The initial, and obtained intermediate and optimal configurations are shown in Figure 6 . We find that the material near the corners are removed and new cavities are generated close to the corners, then the cavities grow to the center of the fixed design domain. We show in Figure 7 the history of the objective function normalized by the perimeter length of all the boundaries.

From Figure 7, we find that the normalized objective function finally converged to $76.5 \%$ of its initial value. Although the history of the area constraint convergence is not shown due to the lack of the space, it is satisfied in the final shape shown in Figure 7. In view of this numerical example, we consider that the present formulation of the topology optimisation with the heat transfer boundary condition and the heat flux objective are specified also on the boundary appearing through the shape updating process has been demonstrated as effective. 


\section{Concluding remarks}

In this paper, a topological derivative expression has been derived for two-dimensional steady-state heat conduction problems accompanying the heat transfer boundary condition and the objective function consisting of heat flux control on the boundary which appears during the optimisation updating process, and its correctness has been verified through an example comparing the topological derivative values calculated based on its analytical expression with those calculated using the finite difference approximation of the topological derivative. The topology optimisation using the derived topological derivative has been implemented to the existing topology optimisation scheme based on the level set approach, and its effectiveness has been demonstrated through a numerical example in which heat transfer boundary condition and objective function to control the heat flux on the boundary emerging during the shape and topology updating process.

\section{References}

[1] Bendsøe, M.P. and Sigmund, O., Topology optimisation-Theory, Methods and Applications, Springer-Verlag Berlin Heidelberg, 2003.

[2] Nguyen, D., Davare, A., Orshansky, M., Chinnery, D., Thompson, B., and Keutzer, K., Minimization of dynamic and static power through joint assignment of threshold voltages and sizing optimization, Proceedings of the 2003 international symposium on Low power electronics and design, ACM, 158-163, 2003.

[3] Sokolowski, J. and Zolesio, J.-P., Introduction to shape optimization, Springer, Berlin Heidelberg, 1992.

[4] Suzuki, K. and Kikuchi, N., A homogenization method for shape and topology optimization, Computer Methods in Applied Mechanics and Engineering, Vol. 93(3), 291-318, 1991.

[5] Bendsøe, M.P. and Kikuchi, N., Generating optimal topologies in structural design using a homogenization method, Computer Methods in Applied Mechanics and Engineering, Vol. 71(2), 197-224, 1988.

[6] Rozvany, G.I.N., Zhou, M. and Birker, T., Generalized shape optimization without homogenization, Structural Optimization, Vol. 4, 250-252, 1992.

[7] Yamada, T., Izui, K., Nishiwaki, S. and Takezawa, A., A topology optimization method based on the level set method incorporating a fictitious interface energy, Computer Methods in Applied Mechanics and Engineering, Vol. 199, 2876-2891, 2010.

[8] Rozvany, G.I.N., Aims, scope, methods, history and unified terminology of computer-aided topology optimization in structural mechanics, Structural and Multidisciplinary Optimization, Vol. 21(2), 90-108, 2001. 
[9] Matsumoto, T., Yamada, T., Takahashi, T., Zheng, C.J. and Harada, S., Acoustic design shape and topology sensitivity formulations based on adjoint method and BEM, Computer Modeling in Engineering \& Sciences (CMES), Vol. 78(2) 77-94, 2011.

[10] Jing, G., Matsumoto, T., Takahashi, T., Isakari, H. and Yamada, T., Topology optimization for 2D heat conduction problems using boundary element method and level set method, Transactions of JASCOME, Vol. 13, 91-96, 2013.

[11] Gouri, D., Lefrançois, E. and Touzot, G., Finite Element Method, John Wiley \& Sons, 2012. 